\title{
Antimicrobial Activities of New Macrocyclic Esters Containing Furan and Thiophene Rings
}

\author{
Hafize Özcan, Anıl Deliorman, Ömer Zaim \\ Department of Chemistry, Faculty of Sciences, Trakya University \\ 22030 Edirne, Turkey \\ hafizeozcan@trakya.edu.tr
}

\section{Extended Abstract}

Cyclic polyethers, commonly known as crown ethers, were first synthesized by Pederson in 1967 [1,2]. Crown ethers were the first synthetic molecules which could, by virtue of their ability to assume a conformation resulting in an electronrich cavity, function as macrocyclic hosts, [3] Prior to 1967 host/guest chemistry was restricted to biologically active hosts, with cyclodextrins being the most common. The oxygen atoms have been replaced by sulfur, [4] nitrogen, [5] and the ethyleneoxy units have been interchanged with heterocycle such as pyridine, furan, thiophene.

We have recently reported the synthesis of [2+2] condensed cyclic amides and investigated antimicrobial activities of these cycloheterophane amides [6]. As a continuation of this work new heterocyclic macrothioesters which contain furan and thiophene rings were synthesized with 2-mercaptoethyl ether and MICs were determined by the microbroth dilution method using the National Committee for Clinical Laboratory Standards (NCCLS) recommendations [7]. For this purposes Gram - Escherichia coli ATCC 25922, Gram - Escherichia coli 0157H7, Gram + Staphylococcus aureus ATCC 25923, Gram - Listeria monocytogenes ATCC 19115, Gram - Salmonella thphimurium ATCC 14028, Gram + Bacillus cereus ATCC 11778 and also Candida albicansATCC 10231 as yeast were used for the investigation of the antimicrobial activity. For comparing antimicrobial activity, Ampicillin, Gentamycin and Amphotericin were used.Serial dilutions were made that furnished a concentration range from 16 to $256 \mu \mathrm{g} / \mathrm{ml}$. Minimum inhibitory values (MIC) were determined at 600nm. As a result macro thioesters showed strong activity againstS. Aureus, Bacillus cereus, Candida albicans and Listeria. After having these results we havesynthesized new heterocyclic macroesters with 2,2'-thiodiethanol and we are studying the antimicrobial activities of the compounds.

\section{References}

[1] F. Diederich, "Complexation of neutral molecules by cyclophane hosts," Angew. Chem. Int. Ed. Engl., vol. 27, pp. 362386, 1988.

[2] C. J. Pedersen, "Cyclic polyethers and their complexes with metal salts," J. Am. Chem. Soc., vol. 89, pp.70177036, 1967.

[3] J. Cram, "The design of molecular hosts, guests, and their complexes," Angew. Chem. Int. Ed. Engl., vol. 27, pp. 1009$1120,1988$.

[4] J. S. Bradshaw and J. Y. K. Hui, "Macrocyclic sulphide syntheses," J. Heterocycl. Chem., vol. 11, pp. 649-674, 1974.

[5] D. H. Busch, "Distinctive coordination chemistry and biological significance of complexes with macrocyclic ligands," Acc. Chem. Res. vol. 11, pp. 392-400, 1978.

[6] N. M. Aghatabay, O. Parali, O. Zaim, Ç. Baydar, and B. Dulger, "Synthesis, structural aspects, antimicrobial activity and ion transportation investigation of four new [2 + 2] condensed 24-membered cycloheterphane peptides," J. Incl. Phenom. Macrocycl. Chem, vol. 79, pp. 415-424, 2014.

[7] National Committee for Clinical Laboratory Standards, Specialty Collection: Susceptibility Testing. SC21-L.M7-A4. NCCLS, Wayne, PA, 1997. 